\title{
Análise in vitro da precisão dimensional do seccionamento e reunião da ferulização de transferentes de moldagem em implantodontia com resina acrlica Dencrilay Pattern
}

\author{
In vitro analysis of the dimensional accuracy of sectioning and reuniting of \\ the ferulization of molding transferents in implantodonty with acrylic resin \\ DencrilayPattern
}

\author{
1 Sergio Barbosa Ribeiro sergiobribeirovr@hotmail.com \\ 2 Cláudio Luís Melo Silva \\ 3 Sérgio Roberto Montoro

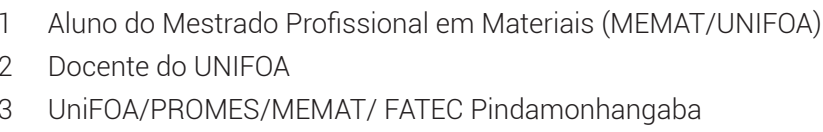

\section{Resumo}

O objetivo deste trabalho foi avaliar in vitro o efeito do seccionamento e reunião da ferulização de transferentes quadrados na precisão dimensional de modelos após a transferência direta de dois implantes, quando a resina Dencrilay Pattern é empregada. Como padrão de aferição dos valores de fenda e qualidade de assentamento sobre os modelos, uma estrutura protética fundida foi confeccionada com emprego de dois pilares UCLAS e distância entre eles de $10 \mathrm{~mm}$. A partir da confecção de um modelo contendo dois implantes, dois grupos para a análise foram formados: o grupo monobloco (GM) e o grupo seccionamento (GS). Um total de 60 aferições $(n=60)$ foram avaliadas com auxílio de microscopia confocal. Complementarmente, uma análise clínica da qualidade de assentamento da estrutura sobre os modelos foi realizada. Os valores médios de fenda e desvio padrão obtidos foram de $31,74 \pm 1,49 \mu \mathrm{m}$ em GC, de 59,83 $\pm 6,40 \mu \mathrm{m}$ em GM e de $41,93 \pm 5,90 \mu \mathrm{m}$ em GS. Seguida da análise estatística ANOVA e Teste de Tukey, diferença estatítica significativa $(P<0,05)$ foi encontrada entre GC e GM, GC e GS e entre GM e GS. O secciomento do monobloco melhorou a qualidade de adaptação da estrutura sobre os análogos além do padrão de assentamento da estrutura protética, apesar da distorção encontrada na resina utilizada.

\section{Palavras-chave}

Implantes dentários. Técnica de impressão. Precisão de impressão. Materiais dentários.

\begin{abstract}
The objective of this work was to evaluate in vitro, the effect of sectioning and meeting of the ferulization of square transferents in the dimensional accuracy of models after the direct transfer of two implants, when the resin Dencrilay Pattern is employed. As a standard for measuring cleft values and nesting quality on the models, a fused prosthetic structure was made using two UCLAS pillars and a distance of $10 \mathrm{~mm}$. From the preparation of a model containing two implants, two groups for the analysis were formed, the monobloc Group (GM) and the sectioning group (GS). A total of 60 measurements ( $n=$ 60 ) were evaluated with the aid of confocal microscopy. In Addition, a clinical analysis of the quality of the structure settlement on the models was performed. The mean values of slit and standard deviation obtained were 31.74 $\pm 1.49 \mu \mathrm{m}$ in $\mathrm{GC}, 59.83 \pm 6.40 \mu \mathrm{m}$ in $\mathrm{GM}$ and $41.93 \pm 5.90$ $\mu \mathrm{m}$ in GS. Followed by statistical analysis ANOVA and Tukey's Test, statistically significant difference $(P<0.05)$ was found between GC and GM, GC and GS, and between GM and GS. The monobloc Secciomento improved the quality of the structure adaptation on the analogs beyond the pattern of the prosthetic structure despite the distortion found in the resin used.
\end{abstract}

\section{Keywords}

Dental implants. Impression techniques. Impression accuracy. Dental materials.

\section{Como você deve citar?}

RIBEIRO, Sergio Barbosa; SILVA, Cláudio Luís Melo; MONTORO, Sérgio Roberto. Análise in vitro da precisão dimensional do seccionamento e reunião da ferulização de transferentes de moldagem em implantodontia com resina acrílica Dencrilay Pattern. Cadernos UniFOA, Volta Redonda, n. 42, p. 115-123, abril. 2020. 
Análise in vitro da precisão dimensional do seccionamento e reunião da ferulização de transferentes de moldagem em implantodontia com resina acrílica Dencrilay Pattern

\section{INTRODUÇÃO}

As principais intercorrências relacionadas à desadaptação marginal e à ausência de passividade em próteses fixas parafusadas sobre múltiplos implantes são predominantemente de natureza mecânica, sendo as mais comuns as fraturas, solturas e perda de torque dos parafusos de retenção (RODRIGUES JÚNIOR et al., 2006). Entende-se como desajuste marginal a fenda presente entre a margem do pilar protético e a restauração protética (RODRIGUES JÚNIOR et al., 2006), e como passividade, a ausência de tensões sobre as estruturas mecânicas e ósseas alveolares, quando a prótese encontra-se assentada sobre os implantes, sem a presença de cargas externas (SAHIN et al., 2001; CAMPI JUNIOR et al., 2010).

Spazzin et al. (2011) e Bacchi et al. (2013) observaram que, na presença de níveis elevados de desajustes marginais, maior concentração de tensões seriam geradas sobre os pilares protéticos, parafusos de retenção prótetica, implantes e o osso cortical, predispondo todo o conjunto mecânico-biológico à ocorrência de alterações deletérias.

Além de encontrar uma correlação positiva entre o padrão de desajuste marginal e a perda de torque dos parafusos de fixação protética, Falcão Filho (2005), com auxílio de microscopia eletrônica de varredura, encontrou alterações significativas nas superfície das roscas desses parafusos de fixação em próteses mais desajustadas.

Percebe-se assim, que a presença de maiores desajustes marginais e a ausência de passividade interferem negativamente na longevidade e sucesso das restaurações implanto-parafusadas sobre implantes. (STÜKER et al., 2005; RODRIGUES JÚNIOR et al., 2006).

Dentre as técnicas de transferência de múltiplos implantes avaliadas pela literatura, aquelas em que os transferentes foram empregados unidos demonstraram superioridade estatística relevante em relação a outras em que os transferentes não foram ferulizados (KIM et al., 2015), principalmente, quando quatro ou mais implantes estavam presentes (BASKAR, 2017). A ferulização preveniria movimento dos transferentes, principalmente os rotacionais durante o arrasto, no momento da remoção da moldeira (GAYATHRIDEVI et al., 2016). A união dos transferentes, de acordo com Moreira (2015), seria uma das etapas mais relevantes no que diz respeito à precisão dimensional final da estrutura protética sobre múltiplos implantes.

No que se refere aos materiais mais frequentemente utilizados na ferulização, a resina acrílica odontológica autopolimerizável Pattern é a mais estudada e empregada em virtude de sua boa estabilidade dimensional (FATIGA et al., 2015; FRANCO et al., 2015, YESHWANTE et al., 2015). Entretanto, todos os materiais que possuem sua constituição baseada em resina sofrem alguma contração e deformação em seu processo de cura, sendo uma das causas dos maiores problemas encontrados na prática clínica, quando se necessita de grande precisão (HORI et al.,2019).

Entre as causas mais comuns relacionadas às alterações dimensionais observadas durante o processo de polimerização, está um inadequado proporcionamento dos constituintes da resina (polímero e monômero). A adição de menores quantidades de polímero (pó), por exemplo, resultaria em maiores alterações dimensionais, sendo a ocorrência delas mais significativas ( $80 \%$ do total) nos primeiros 17 minutos após o preparo (MOJON et al., 1990).

Considerando esse proporcionamento, uma das técnicas mais sensíveis à ocorrência de distorções, e também a mais utilizada pelos profissionais, seria a técnica de inserção da resina com pincel. Tal fato decorreria da inexistência de uma adequada padronização das porções levadas pelo pincel durante a aplicação da resina (FATIGA et al., 2015; FRANCO et al., 2015). Adicionalmente, uma tensão excessiva provocada pela amarração do fio dental aos transferentes poderia acentuar tais consequências (LOPES JÚNIOR et al., 2013). 
Com o intuito de reduzir tais efeitos, alguns procedimentos têm sido estudados e propostos, sendo o principal deles o seccionamento e reunião da ferulização entre os transferentes (YESHWANTE et al., 2015). Cabral \& Guedes (2007) encontraram uma melhora significativa na precisão dos modelos, quando o seccionamento foi realizado 17 minutos após o preparo da resina. Melhora também foi percebida por Vasconcellos et al. (2008), que encontraram uma desadaptação marginal de $70,83 \pm 29,03 \mu \mathrm{m}$, quando a ferulização foi seccionada e reunida após 24 horas, e de 143,25 $\pm 53,48 \mu \mathrm{m}$, quando o seccionamento não foi realizado.

O objetivo deste trabalho foi avaliar in vitro o efeito do seccionamento e reunião da ferulização na precisão dimensional de modelos após a transferência direta de dois implantes, quando a resina Dencrilay Pattern foi empregada.

\section{MATERIAIS E MÉTODOS}

Para a união dos transferentes foi utilizada a resina acrílica odontológica autopolimerizável Dencrilay Pattern (Dencril - Com. e Ind. de Plásticos LTDA - Caieiras- SP - Brasil).

Selecionada a resina, uma estrutura protética metálica fundida em liga de Níquel-Cromo ( $\mathrm{NiCr}$ ) foi confeccionada, utilizando-se dois pilares calcináveis UCLAS Universal II (UN II) de Hexágono Interno (HI) de $4 \mathrm{~mm}$, com cinta metálica antirrotacional (Implacil De Bortoli Mat. Odont. LTDA - São Paulo SP - Brasil) e distância entre os pilares de $10 \mathrm{~mm}$ (Figura 1A), como índice de aferição dos modelos.

Um modelo-mestre (Grupo Controle) foi produzido a partir da confecção de um bloco retangular em resina acrílica autopolimerizável incolor (JET - Artigos Odontológicos Clássicos - São Paulo - SP - Brasil) contendo duas perfurações espaçadas em $10 \mathrm{~mm}$.

Dois implantes cilíndricos de Hexágono Interno UN II de 4mm x 13mm (Implacil De Bortoli Mat. Odont. LTDA - São Paulo - SP - Brasil) foram selecionados, parafusados com torque de $20 \mathrm{Ncm}$ sob a estrutura protética, inseridos nas perfurações do bloco de resina (modelo-mestre) e colados com cola de etilcianoacrilato (Super-bonder - Loctite. Henkel Ltda.). A superfície polida da plataforma cervical dos implantes foi posicionada externamente ao bloco de resina (Figura 1B).

Figura 1 - A- estrutura fundida em NiCr com 10 mm de espaçamento entre os pilares (Padrão); Bmodelo-mestre (controle) confeccionado.

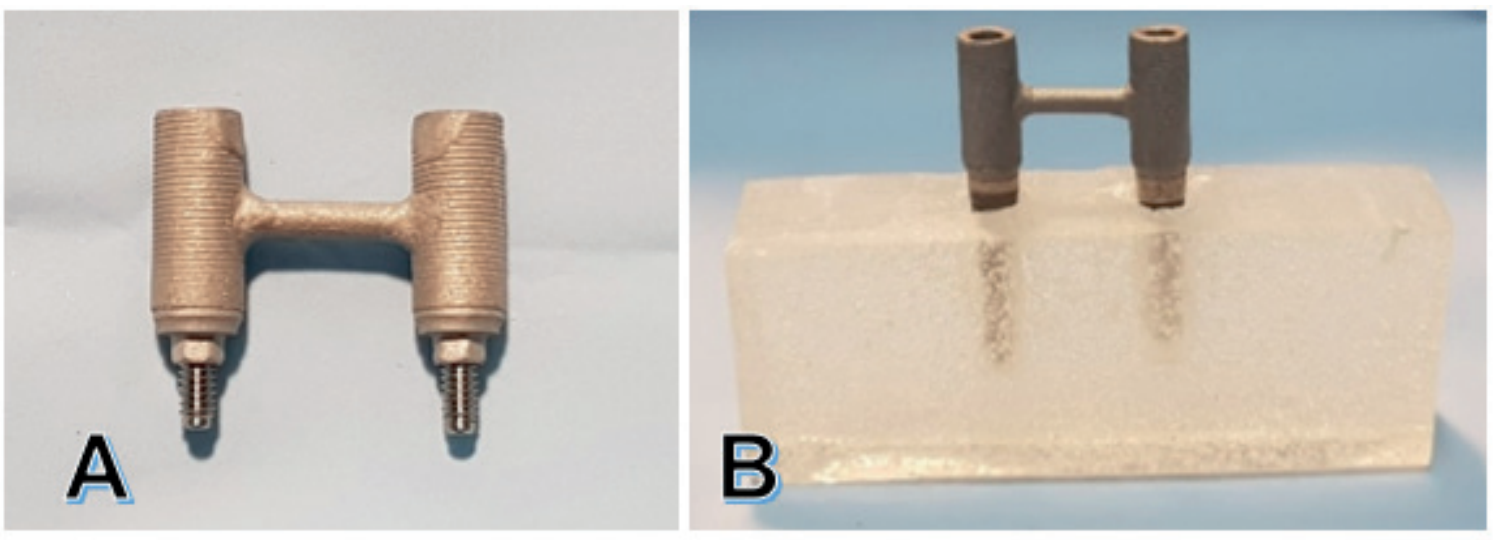

Fonte: autor, 2020. 
Análise in vitro da precisão dimensional do seccionamento e reunião da ferulização de transferentes de moldagem em implantodontia com resina acrílica Dencrilay Pattern

\subsection{Método de Transferência dos Implantes e Grupos para Análise}

\subsubsection{Grupo Monobloco (GM)}

A transferência dos implantes a partir do modelo-mestre foi realizada diretamente com emprego de transferentes quadrados de moldeira aberta $\mathrm{HI}$ de $4 \mathrm{~mm}$ (Implacil De Bortoli Mat. Odont. LTDA - São Paulo - SP - Brasil). Os transferentes foram parafusados sobre os implantes com torque de $10 \mathrm{Ncm}$ e, em seguida, unidos pelo entrelaçamento do fio dental (Colgate Total. Colgate - Palmolive Industrial LTDA.) (Figura 2A) e aplicação da resina Dencrilay Pattern com pincel sobre o fio (Figura 2B).

Figura 2 - A- Análogos parafusados nos implantes do modelo-mestre e entrelaçados por fio dental; B- Resina Pattern aplicada com pincel sobre o fio dental entre os transferentes.
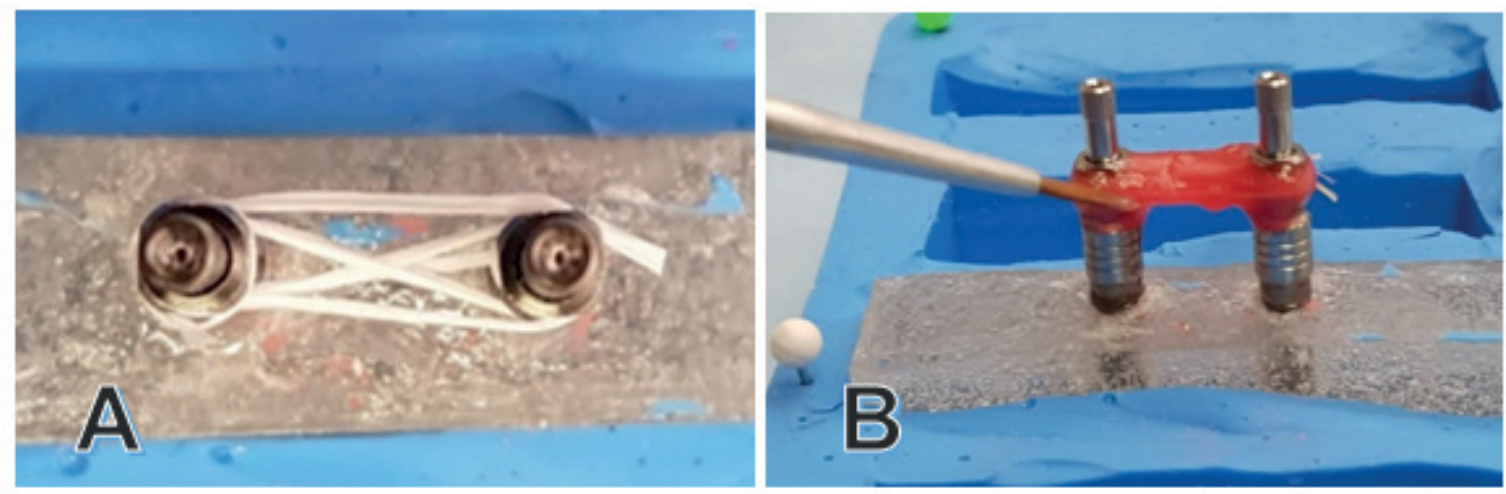

Fonte: autor, 2020.

Finalizada a aplicação da resina sobre o fio dental, um período de 17 minutos aferido com cronômetro foi aguardado para a remoção dos transferentes em monobloco. Em seguida, foram parafusados sobre os análogos $\mathrm{HI}$ de $4 \mathrm{~mm}$ com torque de $10 \mathrm{Ncm}$ e inseridos em uma forma de borracha de silicone industrial PS (Redelease. Barueri - SP) contendo cinco nichos. Gesso especial Tipo IV (Herostone Vigodent S/A Ind e Com-Bonsucesso- RJ - Brasil) foi preparado e vertido com auxílio de um aparelho vibrador de gesso (VH Gold line) sobre os nichos do molde.

Ao fim da presa do gesso, os modelos foram desenformados e levados pra aferição microscópica (Figura 3).

Figura 3 - Modelos de Prova após remoção dos nichos de confecção (GM).
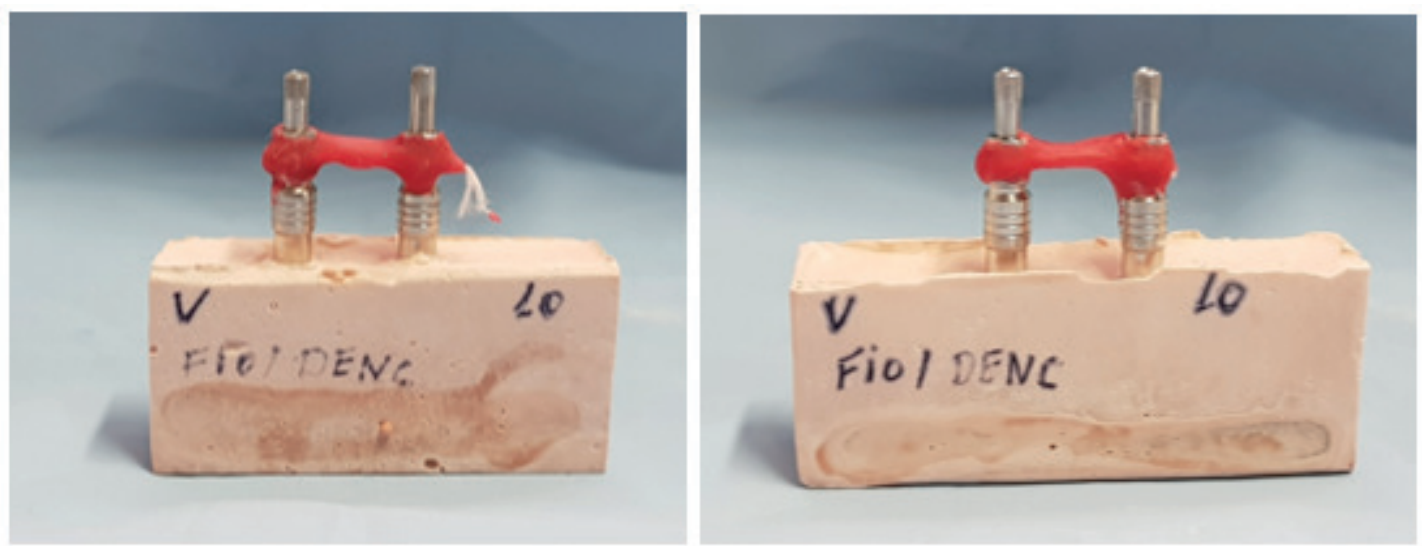

Fonte: autor, 2020. 


\subsubsection{Grupo Seccionamento (GS)}

Finalizada a aferição no microscópio confocal, todos os cinco pares de transferentes unidos foram seccionados com auxílio de discos de carborundum no centro da barra de resina (Figura 4A), reaparafusados com torque de $10 \mathrm{Ncm}$ aos implantes do modelo-mestre e, em seguida, novamente reunidos com a mesma resina, aplicada na interface seccionada (Figura 4B). Após 17 minutos, foi realizada a remoção dos transferentes do modelo.

Figura 4 - A- Seccionamento da ferulização do espécime 5 do grupo monobloco (GM); B- reunião com resina Dencrilay Pattern sobre o modelo-mestre.

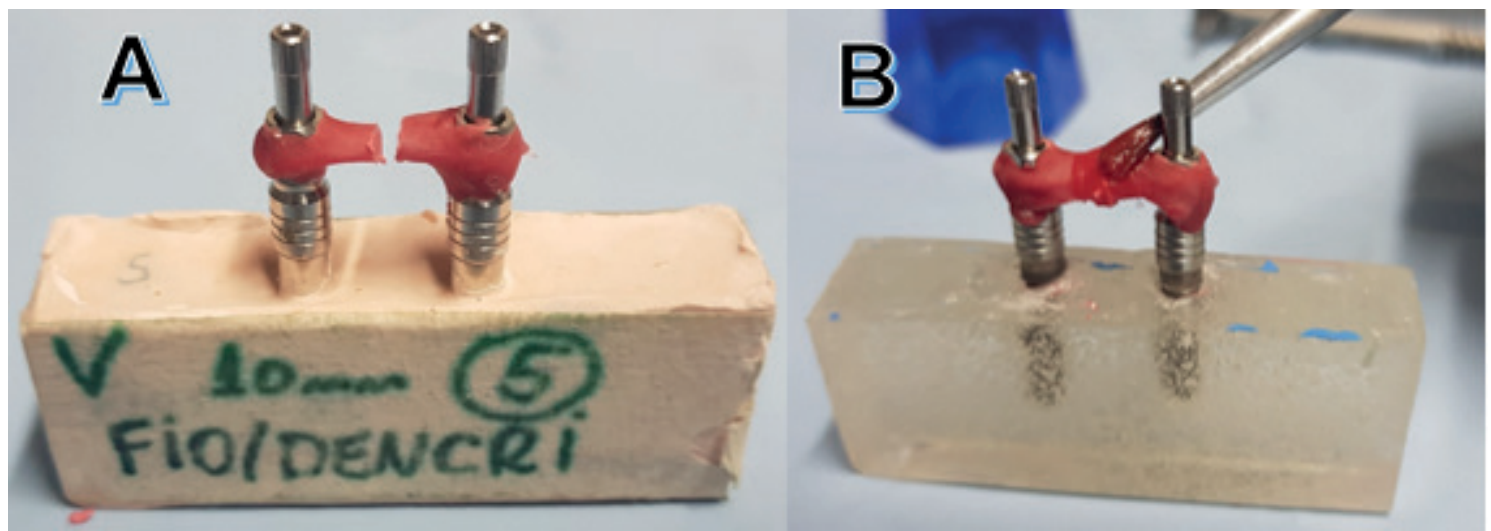

Fonte: autor, 2020.

Os conjuntos de transferentes foram, então, novamente parafusados aos análogos, inseridos no molde de silicone e o gesso vertido sobre o molde. Após o endurecimento completo do gesso, os modelos foram removidos e seguiram novamente à avaliação microscópica.

\subsection{Análise microscópica dos modelos}

Previamente à avaliação microscópica, uma das faces do modelo-mestre e da estrutura protética foi aleatoriamente escolhida e identificada. Em seguida, foram realizadas as aferições no modelo.

Todos os modelos-teste (GM e GS) receberam uma etiqueta de identificação, além de uma numeração de 1 a 5 referente à ordem de avaliação ao microscópio (Figura 4A). Nas aferições, um microscópio confocal Axio CSM 700 com lentes Carl Zeiss com aumento de $110 \mathrm{X}$ foi empregado.

Em seguida, a estrutura protética foi parafusada sobre os modelos com um torquímetro manual (implacil De Bortoli Mat. Odont. LTDA - São Paulo - SP - Brasil), com torque total de $20 \mathrm{Ncm}$. A região mais central e iluminada da amostra ao microscópio foi selecionada, e três leituras da fenda foram registradas por análogo. A unidade de medida apresentada na aferição foi dada em $\mu \mathrm{m}$.

\subsection{Análise clínica do assentamento da estrutura protética}

Além da análise microscópica, foi verificada a facilidade de inserção da estrutura protética sobre os modelos de prova clinicamente. Dois níveis de assentamento foram graduados nessa inspeção: Nível 1: assentamento imediato, sem pressão digital, e Nível 2, assentamento após pressão digital. 
Análise in vitro da precisão dimensional do seccionamento e reunião da ferulização de transferentes de moldagem em implantodontia com resina acrílica Dencrilay Pattern

\section{RESULTADOS E DISCUSSÃO}

De um total de 10 modelos de prova, sendo 5 para o Grupo Monobloco (GM) e 5 para o Grupo Seccionamento (GS), três medidas foram registradas por análogo (direito e esquerdo), totalizando 30 medições por grupo $(n=30)$ e 60 aferições totais $(n=60)$. As médias obtidas no modelo-mestre e nos modelos-teste (GM e GS), desvio padrão e análise clínica de assentamento da estrutura estão listadas nas tabelas 1 e 2 .

Tabela 1 - Média dos valores de fenda em $\mu$ m, desvio padrão no Grupo Controle, Monobloco e Seccionamento.

\begin{tabular}{cccc}
\hline GRUPOS & $\begin{array}{c}\text { Controle } \\
(\mathrm{GC})\end{array}$ & Monobloco & Seccionamento \\
& 31,74 & $(\mathrm{GM})$ & 41,93 \\
\hline Média $(\mu \mathrm{m})$ & & 59,83 & \\
& 1,49 & & 5,90 \\
\hline Desvio padrão & & 6,40 & 30 \\
\hline $\mathbf{N}$ & 30 & $\mathrm{GS})$ \\
\hline
\end{tabular}

Fonte: autor, 2020

Tabela 2 - Valores clínicos de nível de assentamento no grupo controle (GC), Monobloco (GM) e Seccionamento (GS).

\begin{tabular}{|c|c|c|}
\hline Grupo avaliado & Modelo de prova & Nível de assentamento \\
\hline \multirow[t]{3}{*}{ GC } & - & 1 \\
\hline & 1 & 2 \\
\hline & 2 & 2 \\
\hline \multirow[t]{6}{*}{ GM } & 3 & 2 \\
\hline & 4 & 2 \\
\hline & 5 & 2 \\
\hline & MÉDIA & 2 \\
\hline & 1 & 1 \\
\hline & 2 & 1 \\
\hline \multirow[t]{4}{*}{ GS } & 3 & 1 \\
\hline & 4 & 1 \\
\hline & 5 & 1 \\
\hline & MÉDIA & 1 \\
\hline
\end{tabular}

Fonte: autor, 2020

Os valores médios de fenda encontrados no grupo GC, GM e GS foram respectivamente, de 31,74 \pm $1,49 \mu \mathrm{m}, 59,83 \pm 6,40 \mu \mathrm{m}$ e 41,93 $\pm 5,90 \mu \mathrm{m}$, sendo o grupo GM o que apresentou a maior alteração dimensional vertical (fenda) entre os grupos avaliados.

A diferença em $\mu$ m encontrada em relação aos valores de fenda entre os grupos GC e GM foi de 28,25 $\mu \mathrm{m}$. Quando submetidos esses resultados à análise estatística de variância de duas vias ANOVA e ao Teste de Tukey, percebeu-se uma diferença estatística significativa $(P<0,05)$ entre esses dois grupos. Observou-se, assim, como previamente descrito por Hori et al. (2019), a presença de alterações dimensionais resultantes do processo de cura do material resinoso (resina Dencrilay Pattern), resultando em uma dificuldade de obtenção de uma maior precisão dimensional. 
Possivelmente a inexistência de uma adequada padronização das porções (polímero e monômero) levadas pelo pincel durante a aplicação da resina (MOJON et al., 1990; FATIGA et al., 2015; FRANCO et al., 2015) seriam responsáveis pelas maiores alterações dimensionais ocorridas.

Quando o seccionamento e reunião do monobloco foi realizado (grupo GS), observou-se uma melhora estatística significativa em relação aos valores de fenda em comparação ao grupo $G M(P<0,05)$, sendo essa diferença de 18,06 $\mu \mathrm{m}$. Tais resultados corroboraram com as observações de Cabral \& Guedes (2007) e de Vasconcellos et al. (2008) de que o seccionamento da união entre os transferentes resultaria em uma melhora significativa na precisão dos modelos e, consequentemente, em uma melhor adaptação marginal.

Apesar dessa melhora observada, uma diferença estatística foi encontrada entre os grupos GS e o GC. Tal resultado demonstraria uma dificuldade de obtenção de uma boa estabilidade dimensional proporcionada pela associação do emprego da resina Dencrilay Pattern ao método de aplicação com pincel, mesmo em pequenas porções.

Ainda assim, foram considerados os valores de fenda obtidos em outros estudos, como o de Vasconcellos et al. (2008), que encontraram valores de $70,83 \pm 29,03 \mu \mathrm{m}$ após o seccionamento e reunião do monobloco, os resultados obtidos neste estudo demonstraram uma melhor qualidade de adaptação marginal. Esse efeito possivelmente resultou da utilização de componentes UCLA com cinta metálica usinada, que promoveriam uma melhor qualidade de adaptação marginal.

Quanto aos valores observados na análise de assentamento da estrutura protética, no grupo GM, todos os 5 modelos apresentaram uma dificuldade clínica de inserção (Nível 2), indicando assim uma possível ausência de passividade. Esses resultados corroboraram com observações de outros estudos, associando a presença de uma maior desadaptação marginal com ausência de passividade (SPAZZIN et al., 2011; DE BACCHI et al., 2013; FALCÃO FILHO, 2005).

O seccionamento e reunião do monobloco (GS) resultou, além da melhora nos valores de desadaptação marginal, como descrito por Yeshwante et al. (2015), em uma melhora significativa na qualidade de assentamento da estrutura. A estrutura protética foi inserida completamente em todos os modelos sem necessidade alguma de intervenção do operador (Nível 1). Isso demonstrou o efeito positivo do seccionamento e reunião da ferulização em relação à obtenção de passividade. Tal efeito pode resultar clinicamente a longo prazo numa redução importante na ocorrência de efeitos deletérios, sejam eles mecânicos e/ou biológicos, sobre o conjunto prótese-implante-osso alveolar (RODRIGUES JÚNIOR et al., 2006; SPAZZIN et al., 2011; BACCHI et al., 2013).

\section{CONCLUSÃO}

Apesar das limitações inerentes ao estudo, pode-se concluir que:

1. Maior desadaptação marginal e dificuldade de assentamento da estrutura protética foram observadas, quando a resina Dencrilay Pattern foi utilizada para a união em monobloco;

2. O seccionamento e reunião da ferulização resultou em uma melhora significativa nos valores de fenda e na qualidade de assentamento da estrutura protética;

3. Quando do emprego da resina Dencrilay Pattern na ferulização de transferentes de moldagem em condições e métodos semelhantes aos empregados neste estudo, recomenda-se o seccionamento e reunião da ferulização. 
Análise in vitro da precisão dimensional do seccionamento e reunião da ferulização de transferentes de moldagem em implantodontia com resina acrílica Dencrilay Pattern

\section{REFERÊNCIAS}

BACCHI, A. et al. Stress distribution in fixed-partial prosthesis and peri-implant bone tissue with different framework materials and vertical misfit levels: a three-dimensional finite element analysis. Journal of Oral Science, [S.L], v. 55, n. 3, p. 239-244. 2013.

BAIG, Mirza Rustum. Multi-unit implant impression accuracy: A review of the literature. Quintessence international, [S.L], v. 45, n. 1, p.39-51. 2014.

BASKAR, Sushmitha. The Accuracy of Implant Impressions: A Systematic Review. Research Journal of Pharmacy and Technology; Raipur Vol. 10, Ed. 3, Mar 2017, p. 896-902.

CABRAL, L. M.; GUEDES, C. G.. Comparative Analysis of 4 Impression Techniques for Implants. Implant dentistry, [S.L], v. 16, n. 2. 2007.

CAMPI JUNIOR, L. et al. Passividade da prótese sobre implante. Innov Implant J,Biomater Esthet, São Paulo, v. 5, n. 3, p. 53-59, set./dez. 2010.

FATIGA, M. et al. Redes de Bragg utilizadas para mensuração da contração de polimerização de duas resinas acrílicas na moldagem aberta de prótese sobre implantes. Clin Lab Res Den, [S.L], v. 21, n. 3, p. 163-170. 2015.

FRANCO, A. P. G. O. et al. Evaluation of shrinkage polymerization and temperature of diferente acrylic resins used to splinting transfer copings in indirect impression technique. 24th International Conference on Optical Fibre Sensors, [S.L], v. 9634, mar. 2015. Disponível em: <http://proceedings.spiedigitallibrary. org/>. Acesso em: 08 abr. 2018.

GAYATHRIDEVI, S.K; Harshita Gowda; Vaishali K.; Suma. Impression Techniques in Implants. Journal of Dental \& Oro-facial Research, Vol 12 Issue 02 Aug 2016.

HORI, Miki; HORI,Tadasuke; SEKINE Hironao; MIEKI Akimichi; FUJIMOTO Kotaro; KAWAI Tatsushi. Shrinkage characteristics of a novel lower contractile acrylic pattern resin. Dental Materials Journal, p. 1-9, 2019.

KIM, Jee-Hwan; KIM, Kyoung Rok; KIM, Sunjai. Critical appraisal of implant impression accuracies: A systematic review. The Journal of Prosthetic Dentistry, [S.L], v. 114, n. 2, p. 185-192, ago. 2015.

LOPES JÚNIOR, I. et al. Impression Techniques for Multiple Implants: A Photoelastic Analysis. Part II: Comparison of Four Acrylic Resins. Journal of Oral Implantology, [S.L], v. XXXIX, n. 5. 2013.

MOJON, P. et al. Polymerization shrinkage of index and pattern acrylic resins. J Prosthet Dent, [S.L], v. 64, p. 684-688. 1990.

MOREIRA, A. H. J. et al. Accuracy Comparison of Implant Impression Techniques: A Systematic Review. Clinical Implant Dentistry and Related Research, [S.L], v. 17, n. Supplement 2. 2015.

RODRIGUES JUNIOR, W. et al. Assentamento Passivo - Revisão de Literatura. ImplanNews, [S.L], v. 3, n. 1, jan./fev. 2006.

SAHIN S, CEHRELI MC. The significance of passive framework fit in implant prosthodontics: current status. Implant Dent (Baltimore), 2001;10(2):85-92. 
SPAZZIN, A. O. et al. Effects of Horizontal Misfit and Bar Framework Material on the Stress Distribution of an Overdenture-Retaining Bar System: A 3D Finite Element Analysis. Journal of Prosthodontics, [S.L], v. 20, p. 517-522. 2011.

STIMMELMAYR, M. et al. Clinical study evaluating the discrepancy of two different impression techniques of four implants in an edentulous jaw. Clin Oral Invest., Springer-Verlag Berlin Heidelberg, Published Online v. 26, dez. 2012. DOI 10.1007/s00784-012-0885-z.

STÜKER, et al. Passividade da estrutura metálica para próteses fixas implanto-suportadas. Revista Odonto Ciência, Fac. Odonto/PUCRS, v. 20, n. 50, out./dez. 2005.

VASCONCELLOS, D. K. D. et al. Precisão de duas técnicas de esplintagem com resina acrílica de transferentes para próteses sobre implantes. Revista Odonto, São Bernardo do Campo, SP, Metodista, n. 32, jul./dez. 2008.

YESHWANTE, Babita J.; Gaikwad, Sonali Vikas; Baig, Nazish; Patil, Sonali; Shaikh, Wahab A. Comparative evaluation between accuracy of implant impression techniques: A Systematic Review. Journal of Dental and Medical Sciences, Volume 14, Issue 4 Ver. X (Apr. 2015), p. 30-36. 